\title{
МОДЕЛИ И АЛГОРИТМЫ АДАПТИВНОЙ ИНТЕРПРЕТАЦИИ РЕЗУЛЬТАТОВ КОМБИНИРОВАННЫХ ГАЗОГИДРОДИНАМИЧЕСКИХ ИССЛЕДОВАНИЙ ИНТЕЛЛЕКТУАЛЬНЫХ СКВАЖИН
}

\author{
Сергеев Виктор Леонидович', \\ SergeevVL@ignd.tpu.ru \\ Нгуен Тхак Хоай Фыонг, \\ nguyenphuongtpu1512@gmail.com \\ 1 Национальный исследовательский Томский политехнический университет, \\ Россия, 634050, г. Томск, пр. Ленина, 30.
}

Актуальность работы обусловлена необходимостью создания моделей и алгоритмов обработки результатов газогидродинамических исследований интеллектуальных скважин, оснащенных стационарными информационно измерительными системами, позволяющими определять параметры пластов и скважин в процессе проведения испытаний в режиме реального времени. Цель исследования: повышение эффективности и качества интегрированных систем моделей (дебитов, забойных давлений, дополнительных априорных данных и экспертных оценок параметров газовых пластов) и алгоритмов адаптивной идентификации и интерпретации результатов комбинированных газогидродинамических исследований скважин на стационарных, по индикаторной кривой, и нестационарных, по кривой восстановления давления, режимах испытаний.

Методы. Использованы теоретические и практические разработки в области газогидродинамических исследований скважин, системного анализа, идентификации систем, оптимизации функций и линейной алгебры. Для анализа точности и устойчивости моделей и алгоритмов идентификации и интерпретации использовались промысловые данные газодинамических исследований скважин месторождения Тюменской области по индикаторной кривой и кривой восстановления давления, экспертные оценки пластового давления и фильтрационных параметров пласта.

Результаты. Проведен анализ эффективности и качества моделей и алгоритмов идентификации и интерпретации на примерах обработки результатов испытаний нефтяных и газовых скважин по индикаторной кривой и кривой восстановления давления. Показано, что интегрированные системы моделей и алгоритмы адаптивной идентификации и интерпретации позволяют: определять фильтрационные параметры и энергетическое состояние пластов и скважин, число режимов и время завершения исследований в процессе их проведения в режиме реального времени; обеспечить устойчивость и повысить точность оценок проницаемости, пъезопроводности пласта, пластового давления, скин-фактора скважины за счет использования и корректировки дополнительных априорных данных и экспертных оценок пластового давления и фильтрационных параметров пластов; обрабатывать короткие недовосстановленные кривые забойного давления скважины после ее остановки.

\section{Ключевые слова:}

Системный анализ, идентификация, интерпретация, газогидродинамические исследования скважин, индикаторная кривая, кривая восстановления давления, интегрированные системы моделей,

априорная информация, газоконденсатные месторождения.

\section{Введение}

Проведение газогидродинамических исследований скважин (ГДИС) по индикаторной кривой (ИК) и кривой восстановления давления (КВД), а также комбинированных многоциклических ГДИС (ИК-КВД) является необходимым и обязательным мероприятием, поскольку позволяет получить наиболее достоверную информацию о фильтрационных параметрах и энергетическом состоянии пластовых систем, необходимую для создания постоянно действующих моделей, эффективного управления процессами нефтегазодобычи и принятия решений.

В настоящее время в нефтяных и газовых компаниях России преобладают преимущественно традиционные графоаналитические методы и технологии ГДИС, особенностью которых являются [1]:

1. Число режимов исследования по ИК и время завершения исследований на неустановившихся режимах фильтрации по КВД планируется заранее, что часто приводит к большим материальным затратам, связанным с простоями скважин.
2. Процесс интерпретации результатов ГДИС по графоаналитическому методу не позволяет получить параметры пластовых систем в режиме реального времени.

3. Процедура определения режимов течения в процессе проведения гидродинамических исследований путем анализа производной КВД вызывает значительные трудности при интерпретации коротких недовосстановленных КВД, особенно в условиях отсутствия радиального и псевдорадиального режимов течения.

Следует отметить, что с целью сокращения времени исследований были предложены разные модификации традиционных ГДИС, включая широко известные методы монотонно-ступенчатого изменения дебита, такие как изохронный метод, ускоренно-изохронный метод и др. [1-9].

Однако применение традиционных графоаналитических методов и их модификаций при обработке результатов газогидродинамических исследований интеллектуальных скважин, оснащенных стационарными информационно-измерительными системами в режиме реального времени, вызывает значительные трудности, связанные с отме- 
ченными выше проблемами. Здесь необходимы новые технологии, примером которых является метод адаптивной интерпретации ГДИС, модели и алгоритмы идентификации и оптимизации, на основе современных достижений теории систем, системного анализа, идентификации и принятия решений в условиях неопределенности [10-13].

Данная работа является развитием технологии адаптивной идентификации и интерпретации. В ней проводится анализ эффективности и качества интегрированных систем моделей гидродинамических параметров с учетом и корректировкой дополнительных априорных данных, а также рекуррентных и не рекуррентных алгоритмов адаптивной интерпретации результатов комбинированных ГДИС (ИК-КВД).

\section{Интегрированные системы моделей комбинированных ГДИС}

Анализ эффективности и качества алгоритмов адаптивной идентификации и интерпретации комбинированных ГДИС проведем на примерах интегрированной системы моделей (ИСМ) индикаторной кривой Форхгеймера $[14,15]$ с учетом дополнительной информации о пластовом давлении $\bar{p}_{\text {пл, }}$ и коэффициентах фильтрационного сопротивления $\bar{a}_{n}, \bar{b}_{n}[10]$

$$
\left\{\begin{array}{l}
\left(p_{31, n}^{*}\right)^{2}=p_{\text {пл, }, n}^{2}-a_{n} q_{n}-b_{n} q_{n}^{2}+\xi_{n}, \\
h_{\mathrm{\kappa} 1, n} \cdot \bar{p}_{\text {пл }, n}^{2}=p_{\text {пл }, n}^{2}+\eta_{n}, h_{\mathrm{\kappa} 2, n} \cdot \bar{a}_{n}= \\
\quad=a_{n}+v_{n}, h_{\mathrm{к} 3, n} \cdot \bar{b}_{n}=b_{n}+\varepsilon_{n}, n=\overline{1, n_{k}}
\end{array}\right.
$$

и интегрированной системы моделей КВД с учетом дополнительной информации о пластовом давлении $\bar{p}_{\text {пл, }, n}$ и параметрах $\bar{\alpha}_{1, n}, \bar{\alpha}_{2, n}[12]$

$$
\left\{\begin{array}{l}
\left(p_{32, n}^{*}\right)^{2}=\alpha_{1, n}+\alpha_{2, n} \lg \left(t_{n}\right)+\xi_{n}, \\
h_{\mathrm{\kappa} 1, n} \cdot \bar{\alpha}_{1, n}=\alpha_{1, n}+v_{n}, h_{\mathrm{\kappa} 2, n} \cdot \bar{\alpha}_{2, n}= \\
=\alpha_{2, n}+\varepsilon_{n}, h_{\mathrm{\kappa} 3, n} \cdot \bar{p}_{\mathrm{пл}, n}^{2}= \\
=\alpha_{1, n}+\alpha_{2, n} \lg \left(\bar{t}_{r}\right)+\eta_{n}, \quad n=\overline{1, n_{\mathrm{tk}}} .
\end{array}\right.
$$

где $p_{31, n}^{*}$ - фактические значения забойного давления, полученные на разных режимах работы скважины $n ; p_{32, n}^{*}$ - фактические значения забойного давления, полученные в моменты времени $t_{n}$ после остановки скважины; $n_{k}$ - число режимов исследования по индикаторной кривой; $n_{t k}-$ объем данных забойного давления; $\bar{t}_{r}$ - экспертная оценка времени восстановления забойного давления до пластового; $\xi_{n}, \eta_{n}, v_{n}, \varepsilon_{n}$ - случайные величины, представляющие погрешности измерений дебита и забойных давлений скважины, ошибки дополнительных априорных сведений и экспертных оценок.

Отметим, что в моделях (1), (2) число режимов $n_{k}$ и время завершения исследований $t_{k}$ по КВД являются неизвестными параметрами и определяются наряду с неизвестными параметрами $p_{\text {пл, },}^{2}, a_{n}, b$ ИСМ ИК (1) и параметрами $\alpha_{1, n}, \alpha_{2, n}$ ИСМ КВД (2) в процессе их проведения. Введение поправочных коэффициентов $h_{\mathrm{kj}, n}, j=\overline{1,3}$ позволяет организовать процесс корректировки дополнительных данных и экспертных оценок $\bar{p}_{\text {пл, }, 2}^{2}, \bar{a}_{n}, \bar{b}_{n}(1)$ и экспертных оценок $\bar{\alpha}_{1, n}, \bar{\alpha}_{2, n}(2)$ в процессе проведения газогидродинамических исследований и соответственно организовать процесс обучения экспертов.

\section{Алгоритмы адаптивной идентификации}

\section{и интепретации ГДИС}

Решение задачи идентификации комбинированных ГДИС с использованием ИСМ (1), (2) рассмотрим на примере ИСМ КВД (2), представленной в унифицированном матричном виде

$$
\left\{\begin{array}{l}
\mathbf{y}_{n}^{*}=F_{0, n} \boldsymbol{\alpha}_{n}+\boldsymbol{\xi}_{n}, \\
H_{\mathrm{\kappa}, n} \overline{\mathbf{z}}_{n}=F_{a} \boldsymbol{\alpha}_{n}+\boldsymbol{\eta}_{n}, n=\overline{1, n_{t k}},
\end{array}\right.
$$

где $\mathbf{y}_{n}^{*}=\left(\left(p_{32, n}^{*}\right)^{2}, n=\overline{1, n_{t k}}\right)-$ вектор столбец фактических значений квадратов забойных давлений в моменты времени $t_{n} ; F_{0, n}=\left(\varphi_{n}=\left(1, \lg \left(t_{n}\right)\right), n=\overline{1, n_{t k}}\right)-$ матрица модели КВД; $F_{a}=\left[\begin{array}{ll}1, & 0 \\ 0, & 1 \\ 1, \lg \left(t_{r}\right)\end{array}\right]$ - матрица модели дополнительных априорных данных $\overline{\mathbf{z}}_{n}=\left(\bar{\alpha}_{1, n}, \bar{\alpha}_{2, n}, \bar{p}_{\text {пл, },}^{2}\right)-$ вектор дополнительных априорных данных; $\boldsymbol{\alpha}_{n}=\left(\alpha_{1, n}, \alpha_{2, n}\right)$ - вектор столбец неизвестных параметров модели КВД; $H_{\mathrm{k}, n}=\operatorname{diag}\left(h_{\mathrm{k} 1, n}, h_{\mathrm{k} 2, n}, h_{\mathrm{k} 3, n}\right)-$ диагональная матрица параметров корректировки дополнительных данных $\overline{\mathbf{z}}_{n} ; \boldsymbol{\xi}_{n}, \boldsymbol{\eta}_{n}-$ векторы случайных величин.

Процедура адаптивной идентификации ИСМ (3) заключается в решении двух оптимизационных задач по определению параметров модели КВД $\boldsymbol{\alpha}_{n}^{*}=\left(\alpha_{1, n}^{*}\left(\omega_{n}\right), \alpha_{2, n}^{*}\left(\omega_{n}\right)\right)$ и управляющих параметров $\omega_{n}^{*}\left(h_{3, n}^{*}, h_{\mathrm{p}, n}^{*}, h_{\mathrm{\kappa}, n}^{*}\right)[12,13]:$

$$
\begin{gathered}
\boldsymbol{\alpha}_{n}^{*}\left(\boldsymbol{\omega}_{n}\right)=\underset{\boldsymbol{\alpha}_{n}}{\arg \min }\left(\Phi\left(\boldsymbol{\alpha}_{n}, \boldsymbol{\omega}_{n}\right)=\right. \\
\left.=J_{0}\left(\boldsymbol{\alpha}_{n}, h_{3 . n}^{*}\right)+J_{a}\left(\boldsymbol{\alpha}_{n}, h_{\mathrm{p} . n}^{*}, h_{\mathrm{\kappa} . n}^{*}\right)\right), \\
\boldsymbol{\omega}_{n}^{*}=\underset{\omega_{n}}{\arg \min _{0}} J_{0}\left(\boldsymbol{\alpha}_{n}^{*}\left(\boldsymbol{\omega}_{n}\right)\right),
\end{gathered}
$$

где запись $\arg \min _{x} f(x)$ означает точку минимума $x^{*}$ функции $f(x)\left(f\left(x^{*}\right)=\min _{x} f(x)\right) ; \Phi\left(\boldsymbol{\alpha}_{n}, \boldsymbol{\omega}_{n}\right)-$ комбинированный показатель качества, составленный из частного показателей качества модели КВД $J_{0}\left(\boldsymbol{\alpha}_{n}, h_{3, n}^{*}\right)$ и частного показателя качества модели дополнительных априорных данных и экспертных оценок $J_{a}\left(\boldsymbol{\alpha}_{n}, h_{\mathrm{p}, n}^{*}, h_{\mathrm{\kappa}, n}^{*}\right) ; h_{3, n}^{*}, h_{\mathrm{p}, n}^{*}, h_{\mathrm{\kappa}, n}^{*}-$ управляющие параметры забывания для организации процесса адаптивной интерпретации, регуляризации и корректировки дополнительных данных и экспертных оценок [12].

Аналогично (4), (5) формулируется задача оптимизации для ИСМ ИК (1), где

$$
\boldsymbol{\alpha}_{n}^{*}\left(\boldsymbol{\omega}_{n}\right)=\left(\boldsymbol{\alpha}_{1}^{* \prime}\left(\boldsymbol{\omega}_{n}\right)=p_{\text {пл }, n}^{*}, \boldsymbol{\alpha}_{2}^{*}\left(\boldsymbol{\omega}_{n}\right)=a_{n}^{*}, \alpha_{3}^{*}\left(\boldsymbol{\omega}_{n}\right)=b_{n}^{* *}\right),
$$


a $p_{\text {пл, }, n}^{*}, a_{n}^{*}, b_{n}^{*}$ - оптимальные оценки пластового давления и коэффициентов фильтрационного сопротивления.

Процесс адаптивной интерпретации комбинированных ГДИС, с использованием моделей (1), (2) и решением оптимизационных задач $(4),(5)$ состоит из следующих основных этапов:

1. С использованием технологии адаптивной идентификации (4), (5) по КВД первого режима исследований (КВД 1 , рис. 4) получаем данные о параметрах пласта, приведенные в табл. 2 , которые далее используем в качестве дополнительных сведений.

2. Процесс идентификации ИСМ ИК (1) начинаем со второго режима гидродинамических исследований при $n=2$, где в результате решения задач (4), (5) получаем оценки коэффициентов фильтрационного сопротивления $a$ и $b$.

3. После второго режима гидродинамических исследований по ИК и КВД принимается решение о их завершении на основе визуального анализа графика, зависимости оценок параметров пласта и скважины от номера режима исследований и времени (рис. $2,3,8)$ либо по критерию стабилизации оценок, где, например, за момент их завершения $n_{k}$ принимается то значение $n$, при котором выполняется неравенство [11]

$$
\left|\left(\alpha_{j, n}^{*}-\alpha_{j, n-1}^{*}\right) / \alpha_{j, n}^{*}\right| \leq \varepsilon_{j}, j=\overline{1, m}, i=1,2,3, \ldots,
$$

$\varepsilon_{j}$ - заданная точность. В противном случае переходим к третьему, четвертому и т. д. режиму исследований до выполнения критерия стабилизации оценок.

Следует отметить, что приведенная выше схема интерпретации комбинированных ГДИС в отличие от традиционных схем дает возможность принимать решение о завершении исследований в процессе их проведения без участия квалифицированного интерпретатора.

\section{Результаты идентификации и интерпретации ГдИС}

Результаты идентификации и интерпретации комбинированных ГДИС газоконденсатного месторождения Тюменской области приведены на рис. 1-8 и в табл. 2.

Исходные данные для результатов интерпретация скважин № 1180, 1041, приведенные на рис. 5 (линия 1), рис. 7 (линия 1), и в табл. 1.

На рис. 1, 2 приведены оценки коэфрициентов фильтрационного сопротивления ИСМ (1) $a_{n}^{*}=\alpha_{2}^{*}\left(\omega_{n}^{*}\right)$, $b_{n}^{*}=\alpha_{3}^{*}\left(\omega_{n}^{*}\right)$, полученные с использованием исходных данных дебитов и забойных давлений скважины № 1180 на трех режимах исследований (табл. 1). Для решения оптимизационной задачи (4) использован комбинированный показатель качества, состоящий из квадратичного показателя качества модели ИК и квадратичного показателя качества экспертной оценки $\bar{\alpha}_{1, n}=\bar{p}_{\text {пл,n }}^{2}$ квадрата пластового давления

$$
\begin{gathered}
\Phi\left(\alpha_{n}, h_{3, n} h_{\mathrm{p}, n}, h_{\mathrm{\kappa}, n}\right)= \\
=\left\|\mathbf{y}_{n}^{*}-F_{0, n} \alpha_{n}\right\|_{K\left(h_{3, n}\right)}^{2}+h_{\mathrm{p}, n}\left(h_{\mathrm{\kappa}, n} \bar{\alpha}_{1, n}-\alpha_{1, n}\right)^{2} .
\end{gathered}
$$

\begin{tabular}{|c|c|c|}
\hline \multirow{3}{*}{$\begin{array}{l}\text { Параметры пласта и скважины } \\
\text { Reservoir and well parameters }\end{array}$} & \multirow{2}{*}{\multicolumn{2}{|c|}{\begin{tabular}{|l} 
Значение/Value \\
Скважина/Well
\end{tabular}}} \\
\hline & & \\
\hline & 1180 & 1041 \\
\hline \begin{tabular}{|l} 
Пластовая температура \\
Reservoir temperature $T_{\text {пл, }}{ }^{\circ} \mathrm{K}$
\end{tabular} & 297,85 & 353,9 \\
\hline Радиус скважины/Well radius $r_{c,}$ м (m) & 0,108 & 0,108 \\
\hline $\begin{array}{l}\text { Атмосферное давление } p_{\mathrm{cm}}, \mathrm{kгc} / \mathrm{cm}^{2} \\
\text { Pressure at standard condition } p_{\mathrm{cm}},\left(\mathrm{kgf} / \mathrm{cm}^{2}\right)\end{array}$ & 1,033 & 1,033 \\
\hline $\begin{array}{l}\text { Температура при нормальных условиях } \\
\text { Standard condition temperature }\left(+20^{\circ} \mathrm{C}\right) T_{\mathrm{cm}},{ }^{\circ} \mathrm{K}\end{array}$ & 293 & 293 \\
\hline \begin{tabular}{|l|} 
Коэффициент сжимаемости газа \\
при пластовых условиях z \\
Compressibility of gas under reservoir conditions z
\end{tabular} & 0,963 & 0,879 \\
\hline $\begin{array}{l}\text { Коэффициент общей сжимаемости газа } \\
\text { Total compressibility } c_{t} \text { с см²/ } / \text { кс }\left(\mathrm{cm}^{2} / \mathrm{kgf}\right) \\
\end{array}$ & 0,0452 & 0,00387 \\
\hline Пористость/Porosity m, д. е & 0,32 & 0,113 \\
\hline \begin{tabular}{|l} 
Эффективная мощность пласта \\
Formation effective thickness $h, \mathrm{~m}(\mathrm{~m})$
\end{tabular} & 57,2 & 168,2 \\
\hline $\begin{array}{l}\text { Динамическая вязкость } \\
\text { Gas viscosity } \mu, \text { сП (ср) }\end{array}$ & 0,0117 & 0,02 \\
\hline $\begin{array}{l}\text { Дебит скважины на режиме } 1 \\
\text { Well flow rate at stage } 1 \text { q0,1, тыс м³/сут } \\
\text { (thousand } \mathrm{m}^{3} / \mathrm{D} \text { ) } \\
\end{array}$ & 143,89 & 280,56 \\
\hline $\begin{array}{l}\text { Дебит скважины на режиме } 2 \\
\text { Well flow rate at stage } 2 \text { q0,2, тыс м³/сут } \\
\text { (thousand } \mathrm{m}^{3} / \mathrm{D} \text { ) }\end{array}$ & 71,08 & 153,56 \\
\hline $\begin{array}{l}\text { Дебит скважины на режиме } 3 \\
\text { Well flow rate at stage } 3 \text { q0,3, тыс м³/сут } \\
\text { (thousand } \mathrm{m}^{3} / \mathrm{D} \text { ) } \\
\end{array}$ & 104,41 & 209,39 \\
\hline $\begin{array}{l}\text { Дебит скважины на режиме } 4 \\
\text { Well flow rate at stage } 4 q_{0,4} \text { тыс м³ } / \text { сут } \\
\text { (thousand } \mathrm{m}^{3} / \mathrm{D} \text { ) } \\
\end{array}$ & 126,06 & 250,13 \\
\hline $\begin{array}{l}\text { Экспертная оценка времени } \\
\text { восстановления забойного давления } \bar{t}_{r,} 4 \\
\text { Expert estimate of time for pressure build up } \\
\text { curve fully recovers } \bar{t}_{r,} \text { hour }\end{array}$ & 3 & 72 \\
\hline $\begin{array}{l}\text { Экспертная оценка пластового давления } \\
\text { Expert estimate of reservoir pressure } \bar{p}_{\text {пл, }} \mathrm{k} г \mathrm{c} / \mathrm{cm}^{2} \\
\left(\mathrm{kgf} / \mathrm{cm}^{2}\right)\end{array}$ & 23,8 & 262 \\
\hline $\begin{array}{l}\text { Экспертная оценка проводимости пласта } \\
\text { xpert estimate of reservoir conductivity } \bar{\sigma}, \\
\text { Д.м/сП (D.m/cp) }\end{array}$ & 2100 & 4,5 \\
\hline
\end{tabular}

Таблица 1. Исходные данные для скважин № 1180 и 1041 Table 1. Initial data of wells 1180 and 1041

В данном случае оптимизационная задача (4), по аналогии с [13], сводится к решению систем линейных алгебраических уравнений (СЛУ) вида

$$
\begin{aligned}
& \left(F_{0, n}^{T} K\left(h_{3, n}\right) F_{0, n}+h_{\mathrm{p}, n} \mathbf{I}\right) \boldsymbol{\alpha}_{n}= \\
= & \left(F_{0, n}^{T} K\left(h_{3, n}\right) \mathbf{y}_{n}^{*}+h_{\mathrm{p}, n} h_{\mathrm{\kappa}, n} \bar{\alpha}_{1, n}\right),
\end{aligned}
$$

где запись $\|X\|_{W}^{2}$ означает квадратичную форму $X^{T} W X ; \mathbf{y}_{n}^{*}=\left(\left(p_{31, n}^{*}\right)^{2}, n=\overline{1, n_{t k}}\right)^{T}-$ вектор столбец фактических значений квадратов забойного давления, полученных на разных режимах работы скважины; $F_{0, n}=\left(\phi_{n}=\left(1, q_{n}, q_{n}^{2}\right), n=1, n_{k}\right)-$ матрица ИСМ (1); $\boldsymbol{\alpha}_{n}=\left(\alpha_{1, n}=p_{\text {пा, }, n}^{2}, \alpha_{2, n}=a_{n}, \alpha_{3, n}=b_{n}\right)^{T}-$ вектор неизвестных значений параметров модели (1); $p_{\text {пл, },}, \bar{p}_{\text {пл, }, n}-$ начальное пластовое давление и его экспертная оценка; $K\left(h_{3, n}\right)=\operatorname{diag}\left(\exp \left((n-i) / h_{3, n}\right), n=\overline{1, n_{k}}, i=\overline{1, n_{k}-1}\right)-$ диагональная матрица значений весовой функции 
$w\left(x / h_{3}\right)$ с параметром забывания $h_{3, n} ; \mathrm{I}-$ единичная матрица.

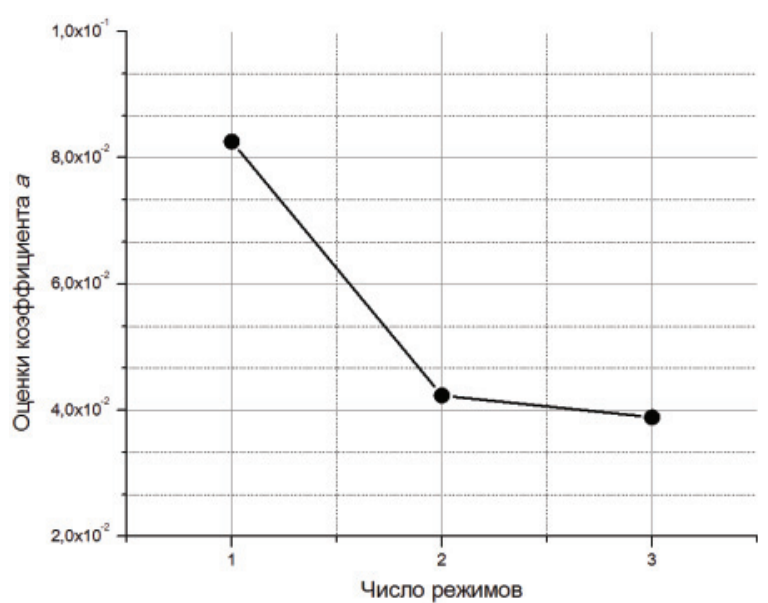

Рис. 1. Оценки коэффициента сопротивления а, $\left(\text { кгс } / \mathrm{CM}^{2}\right)^{2} /$ (тыс. $\mathrm{M}^{3} /$ Сут)

Fig. 1. Estimates of flow coefficient $a_{1}\left(\mathrm{kgf} / \mathrm{cm}^{2}\right)^{2} /($ thousand $\left.\mathrm{m}^{3} / \mathrm{D}\right)$

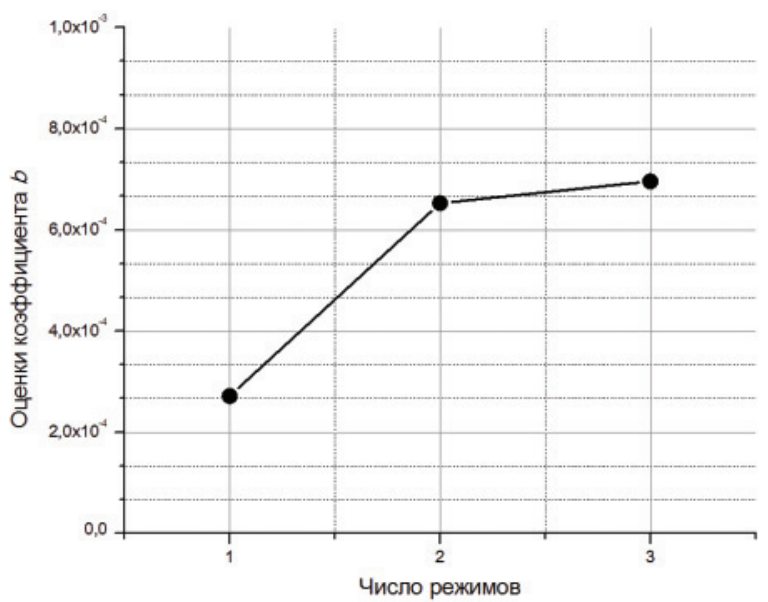

Рис. 2. Оценки коэффициента сопротивления b, $\left.\left(\text { кгс } / \mathrm{CM}^{2}\right)^{2}\right)$ $\left(\text { тыс. } \mathrm{M}^{3} / \mathrm{CYT}\right)^{2}$

Fig. 2. Estimates of flow coefficient $b,\left(\mathrm{kgf} / \mathrm{cm}^{2}\right)^{2} /$ (thousand $\left.\mathrm{m}^{3} / \mathrm{D}\right)^{2}$

На рис. 3 приведены оценки проводимости пласта скважины № 1180 по КВД четвертого режима исследований (рис. 5 КВД 4) без корректировки $\left(h_{\mathrm{k}, n}=1\right)$ экспертных оценок проводимости пласта $\bar{\sigma}_{n}=2100$ Д.м $/ \mathrm{c} \Pi$ и $\bar{\sigma}_{n}=1400$ Д.м/сП, заданных с ошибками $\pm 20 \%$ (линии 3,4 ) и с корректировкой экспертных оценок при $h_{\mathrm{k}, n}=h_{\mathrm{k}, n}^{*}$ (линии 1,2$)$ :

$$
\sigma_{n}^{*}=\frac{42,4 q_{0,4} T_{\text {пл }} p_{\text {ст }} z}{T_{\text {ст }} \alpha_{2, n}^{*}\left(\omega_{n}^{*}\right)},
$$

где значения $q_{0,4}, T_{\text {пл }}, p_{\text {ст }}, T_{\text {ст }}$ приведены в табл. 1. Оценки параметра $\alpha_{2, n}^{*}\left(\omega_{n}^{*}\right)(8)$ определялись по аналогии с (3), (6), (7) с использованием комбинированного показателя качества, состоящего из квадратичного показателя качества модели КВД и квадратичного показателя качества экспертной оценки проводимости пласта $\bar{\sigma}_{n}$

$$
\begin{gathered}
\Phi\left(\alpha_{n}, h_{3, n} h_{\mathrm{p}, n}, h_{\mathrm{K}, n}\right)= \\
=\left\|\mathbf{y}_{n}^{*}-F_{0, n} \boldsymbol{\alpha}_{n}\right\|_{K\left(h_{3, n}\right)}^{2}+h_{\mathrm{p}, n}\left(h_{\mathrm{K} n} \bar{\sigma}_{n}-\sigma_{n}\right)^{2},
\end{gathered}
$$

путем решения СЛУ вида

$$
\begin{aligned}
& \left(F_{0, n}^{T} K\left(h_{3, n}\right) F_{0, n}+h_{\mathrm{p}, n} \mathbf{I}\right) \boldsymbol{\alpha}_{n}= \\
= & \left(F_{0, n}^{T} K\left(h_{3, n}\right) \mathbf{y}_{n}^{*}+h_{\mathrm{p}, n} h_{\mathrm{\kappa}, n} \bar{\sigma}_{n}\right),
\end{aligned}
$$

где $\mathbf{y}_{n}^{*}, F_{0, n}$ определены в (3),

$$
K\left(h_{3, n}\right)=\operatorname{diag}\left(\exp \left(\left(t_{n}-t_{i}\right) / h_{3 n}\right), n=\overline{1, n_{t k}}, i=\overline{1, n_{t k}-1}\right)
$$

- диагональная матрица весовых функций.

Решение оптимизационной задачи (5) для определения управляющих параметров $h_{3, n}, h_{\mathrm{k}, n}, h_{\mathrm{p}, n}$ в (6), (7), (9) (10), по аналогии с [13], сводилось к последовательному решению одномерных оптимизационных задач по определению параметра регуляризации $h_{\mathrm{p}, n}^{*}$, параметра корректировки $h_{\mathrm{\kappa}, n}^{*}$ и параметра забывания $h_{3, n}^{*}$ с использованием метода дихотомии [16].

Следует отметить, что управляющие параметры $h_{3, n}, h_{\mathrm{k}, n}, h_{\mathrm{p}, n}$ в (7), (10) также могут определить методом деформированного многогранника [17].

В табл. 2 приведены оценки проводимости $\sigma_{n}^{*}$ (8), пъезопроводности $\chi_{n}^{*}$, пластового давления $p_{\text {пр, } n}^{*}$ и инстинного скин-фактора $S_{0}^{*}$

$$
\begin{gathered}
\chi_{n}^{*}=\frac{\sigma_{n}^{*}}{h m c_{t}}, p_{\text {пл, }, n}^{*}=\sqrt{\alpha_{1, n}^{*}\left(\omega_{n}^{*}\right)+\alpha_{2, n}^{*}\left(\omega_{n}^{*}\right) \lg \left(\bar{t}_{r}\right)}, \\
S_{0}^{*}=\ln \left(\frac{r_{\tilde{n}}}{r_{\text {пр, }, n}^{*}}\right),
\end{gathered}
$$

полученные по КВД трех газовых скважин на разных режимах их работы, где $\alpha_{1, n}^{*}\left(\omega_{n}^{*}\right), \alpha_{2, n}^{*}\left(\omega_{n}^{*}\right)-$ оценки параметров модели КВД (2), (3), полученные путем решения СЛУ (10); $h, m, c_{t}, r_{c}$ приведены в табл. 1. Приведенный радиус скважины $r_{n p, n}^{*}$ в (11) определялся из уравнения

$$
\alpha_{1, n}^{*}\left(\omega_{n}^{*}\right)=p_{32,0}^{2}+\alpha_{2, n}^{*}\left(\omega_{n}^{*}\right) \lg \left(\frac{2,25 \chi_{n}^{*}}{r_{\text {mp }, n}^{2}}\right)+b_{n}^{*} q_{0, n}^{2},
$$

следующего из модели КВД для однородно пористого пласта (2) [1]. Здесь $p_{32,0}^{2}-$ квадрат пластового давления в момент остановки скважины $t_{0}$; $b_{n}^{*}=\alpha_{3}^{*}\left(\omega_{n}^{*}\right)$ - оценка коэффициента фильтрационного сопротивления, полученная из решения СЛУ (7).

Анализ результатов, приведенных на рис. 2, 3 и в табл. 2, показывает, что оценки фильтрационных параметров пласта, проводимости, пъезопроводности, пластового давления и скин-фактора скважины, полученные методом адаптивной интерпретации (АИ), начиная со второго режима исследований, практически не уступают оценкам на последующих режимах, а также приближениям с использованием программы Saphir, что позволяет сократить время проведения комбинированных ГДИС и, следовательно, время простоя скважин.

Обработка большого объема данных о забойном давлении скважины с решением системы линейных уравнений вида (7), (10) затрудняет процесс 
интерпретации комбинированных ГДИС в режиме реального времени. В этой связи на основе рекуррентных алгоритмов [18, 19], в работе предлагается параметры модели ИСМ КВД (3) определять с использованием рекуррентного алгоритма без решения СЛУ и обращения матрицы [13].

$$
\begin{gathered}
\boldsymbol{\alpha}_{n}^{*}=\boldsymbol{\alpha}_{n-1}^{*}+\Gamma_{n} \boldsymbol{\varphi}_{n}^{T} w_{n}\left(h_{3, n}\right)\left(y_{n}^{*}-\boldsymbol{\varphi}_{n} \boldsymbol{\alpha}_{n-1}^{*}\right), \\
\Gamma_{n}=\Gamma_{n-1}-\frac{\Gamma_{n-1} \boldsymbol{\varphi}_{n}^{T} \boldsymbol{\varphi}_{n} \Gamma_{n-1}}{w_{n}^{-1}\left(h_{3, n}\right)+\boldsymbol{\varphi}_{n} \Gamma_{n-1} \boldsymbol{\varphi}_{n}^{T}}, n=1,2,3, \ldots,
\end{gathered}
$$

$\boldsymbol{\varphi}_{n}=\left(1, \lg \left(t_{n}\right)\right)-$ вектор строка; $\boldsymbol{\alpha}_{0}^{*}=h_{\mathrm{K}} \overline{\mathbf{z}}_{0}-$ вектор начальных оценок параметров.

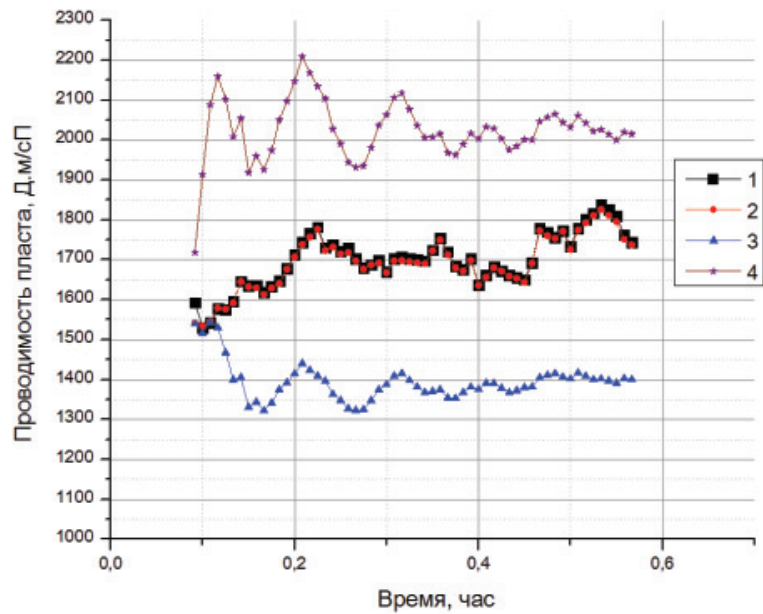

Рис. 3. Оценки проводимости пласта скважины № 1180 с корректировкой (линии 1, 2) и без корректировки экспертных оценок проводимости пласта (линии 3,4$)$

Fig. 3. Estimates of reservoir conductivity of well 1180 with (line 1, 2) and without (line 3, 4) correction of expert estimates of reservoir conductivity

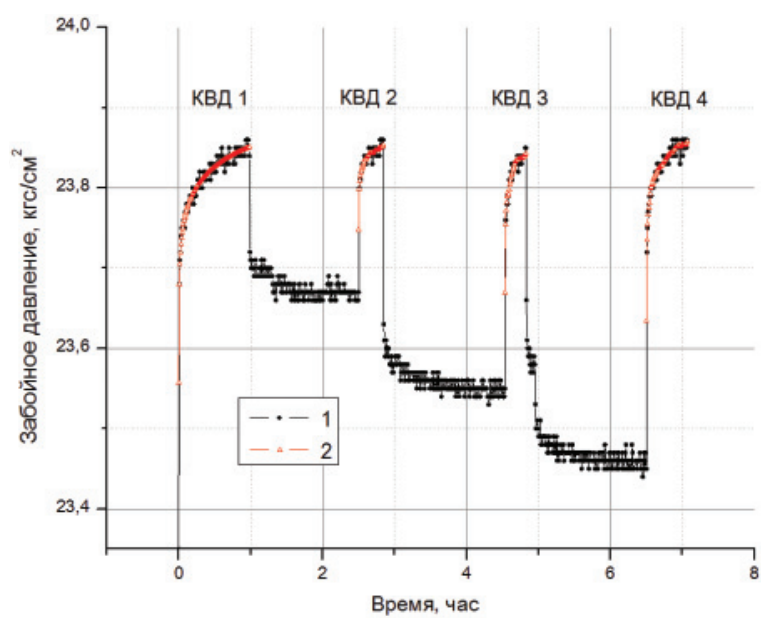

Рис. 4. Исходные (линия 1) и восстановленные (линия 2) значения забойного давления комбинированных ГДИС скважины № 1180 на трех режимах исследований

Fig. 4. Actual (curve 1) and matching (curve 2) data of bottomhole pressure of combined gas well test number 1180 with three test stages
Таблица 2. Оценки параметров пласта и скважины

\begin{tabular}{|c|c|c|c|c|c|c|c|}
\hline \multirow[b]{2}{*}{$\stackrel{\oplus}{\cup} \overline{\bar{d}}$} & \multirow[b]{2}{*}{ 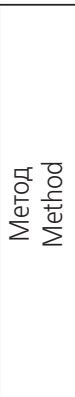 } & \multirow[b]{2}{*}{ 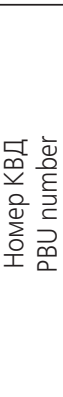 } & \multirow[b]{2}{*}{ 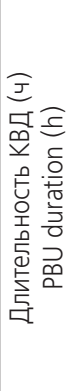 } & \multicolumn{4}{|c|}{$\begin{array}{c}\text { Оценка параметров пласта и скважины } \\
\text { Estimates of reservoir } \\
\text { and well parameters }\end{array}$} \\
\hline & & & & 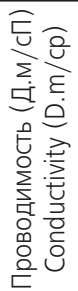 &  & 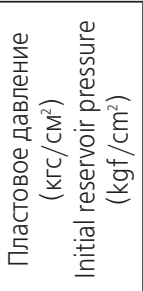 &  \\
\hline \multirow{5}{*}{1124} & \multirow{4}{*}{$\begin{array}{c}\mathrm{A} И \\
\mathrm{Al}\end{array}$} & 1 & 1,35 & 315,7 & 478 & 23,69 & $-1,38$ \\
\hline & & 2 & 0,48 & 292,4 & 443 & 23,59 & $-1,61$ \\
\hline & & 3 & 0,78 & 331,3 & 506 & 23,64 & $-1,36$ \\
\hline & & 4 & 1,36 & 354,6 & 537 & 23,69 & $-1,31$ \\
\hline & Saphir & & & 335,2 & 508 & 23,65 & $-1,22$ \\
\hline \multirow{5}{*}{1125} & \multirow{4}{*}{$\begin{array}{c}\mathrm{A} И \\
\mathrm{Al}\end{array}$} & 1 & 1,04 & 565,1 & 564 & 22,41 & $-1,88$ \\
\hline & & 2 & 0,48 & 547,8 & 551 & 22,23 & $-1,41$ \\
\hline & & 3 & 0,50 & 559,3 & 560 & 22,39 & $-1,32$ \\
\hline & & 4 & 0,58 & 604,2 & 609 & 22,51 & $-1,34$ \\
\hline & Saphir & & & 604,3 & 613 & 22,51 & $-1,26$ \\
\hline \multirow{5}{*}{1180} & & 1 & 0,98 & 1682,4 & 2033 & 23,99 & $-3,08$ \\
\hline & & 2 & 0,34 & 1806,6 & 2183 & 23,94 & $-1,72$ \\
\hline & & 3 & 0,29 & 1692,3 & 2045 & 23,97 & $-2,59$ \\
\hline & & 4 & 0,57 & 1745,8 & 2109 & 23,99 & $-2,61$ \\
\hline & Saphir & & & 1712,1 & 2069 & 23,98 & $-3,01$ \\
\hline
\end{tabular}

Table 2. Estimates parameters of reservoir and well

Определение оптимальных значений управляющих параметров забывания, регуляризации и корректировки ввиду большого объема вычислений предлагается, по аналогии с [13], определять не на всем диапазоне забойных давлений, а в определенные моменты времени $t_{n(k)}, k=1,2,3 \ldots$, на соответствующих участках, например через $3-5$ часов газогидродинамических исследований.

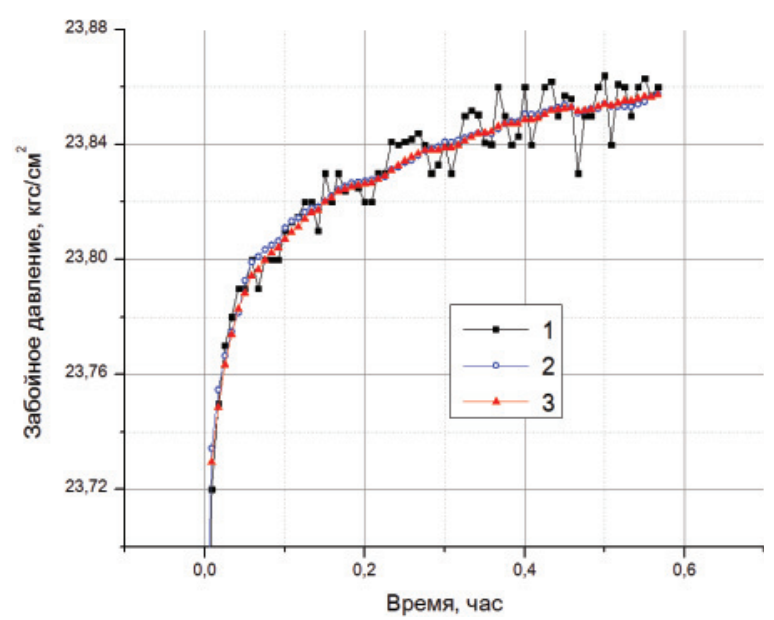

Рис. 5. Исходные (линия 1) и восстановленные (линии 2, 3) значения КВД скважины № 1180

Fig. 5. Actual (curve 1) and matching (curve 2, 3) data of bottomhole pressure well 1180

На рис. 5 приведены исходные (линия 1) и восстановленные значения КВД четвертого режима ис- 
следований (рис. 4 КВД-4), где оценки параметров проводимости (8) определялись путем решения СЛУ (10) (линия 2) и по рекуррентным алгоритмам (12) (линия 3) при $\boldsymbol{\alpha}=\overline{\mathbf{z}}_{0}=\left(0, \bar{\alpha}_{2,0}=2100\right.$ Д·м/сП).

На рис. 6 приведены оценки проводимости пласта, полученные путем решения СЛУ (10) (линия 1), и с использованием рекуррентных алгоритмов (12) (линия 2). Линией 3 изображены оценки проводимости пласта, полученные традиционным методом наилучшего совмещения, который следует из (10) при $h_{p, n}=0$.

Рекуррентные оценки проводимости пласта не уступают по точности нерекуррентным оценкам, что подтверждает целесообразность их практического использования (рис. 5,6$)$.

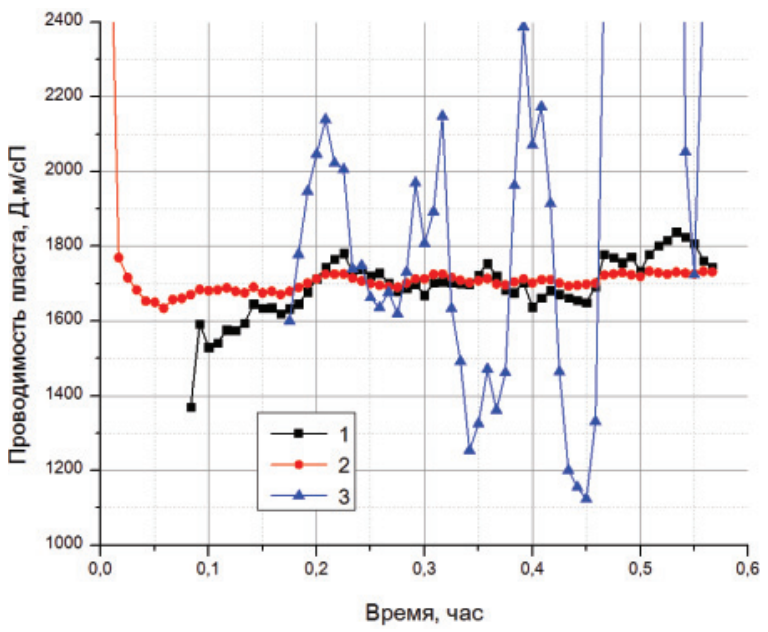

Рис. 6. Оценки проводимости пласта скважины № 1180

Fig. 6. Estimates of reservoir conductivity of well 1180

На рис. 7 приведены исходные (линия 1) и восстановленные значения КВД скважины 1041, где оценки параметров проводимости (8) определялись адаптивным методом интерпретации путем решения СЛУ (10) (линия 2).

На рис. 8 приведены оценки проводимости пласта скважины № 1041, полученные адаптивным методом интерпретации путем решения СЛУ (10) (линия 1), адаптивным методом наилучшего совмещения при $h_{p, n}=0$ (линия 2) и адаптивным методом наилучшего совмещения с регуляризацией при $h_{p, n}=h_{p, n}^{*}$ (линия 3) [20].

Стабилизация оценок проводимости пласта, полученных методом адаптивной интерпретации с учетом и корректировкой априорной информации (рис. 8, линия 1), наступает в пределах 3-5 часов, что значительно раньше по сравнению с оценками, полученными адаптивным метом наилучшего совмещения с регуляризацией (рис. 8 линия 3), газогидродинамических исследований.

Результаты интерпретации, приведенные на рис. 6,8 (линия 1), отражают устойчивость оценок проводимости пласта, полученных методом адаптивной интерпретации с учетом и корректировкой априорной информации, и их достаточно быструю сходимость.

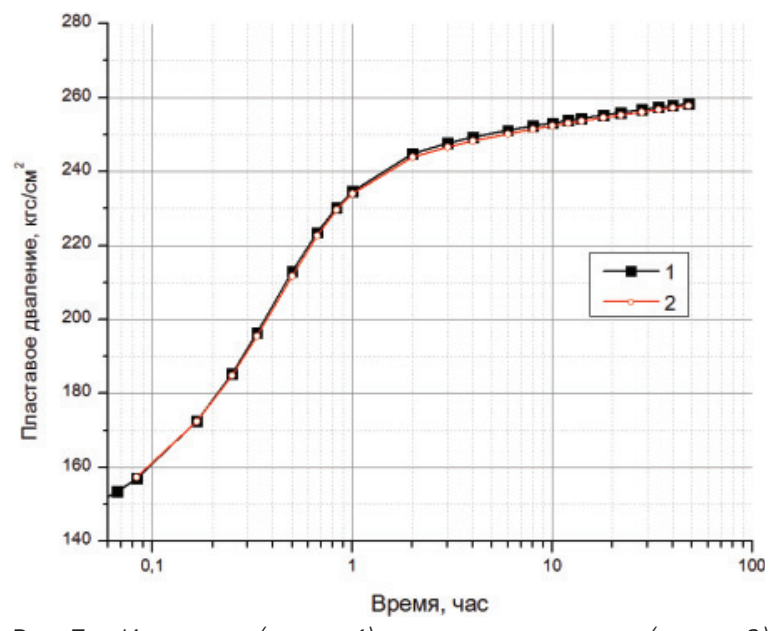

Рис. 7. Исходные (линия 1) и восстановленные (линия 2) значения КВД скважины № 1041

Fig. 7. Actual (curve 1) and matching (curve 2) data of bottomhole pressure well 1041

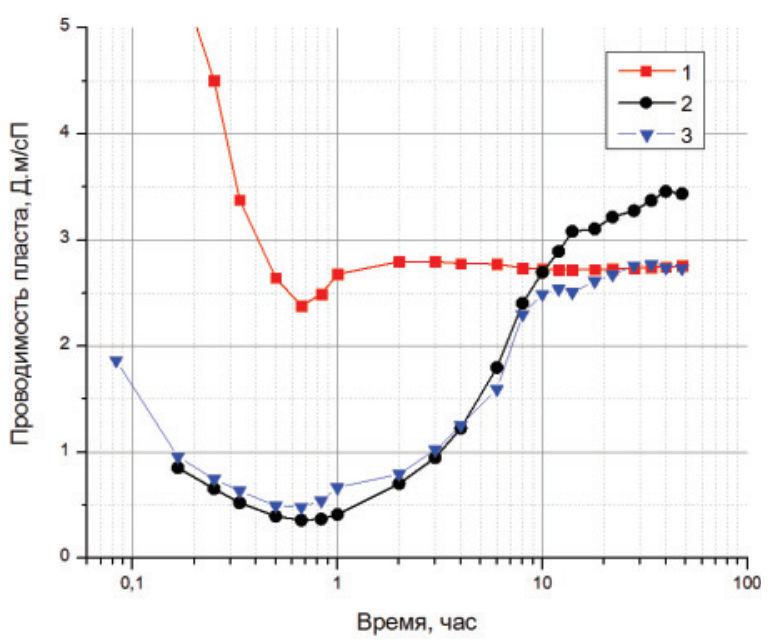

Рис. 8. Оценки проводимости пласта скважины № 1041

Fig. 8. Estimates of reservoir conductivity of well 1041

\section{Выводы}

Проведен анализ эффективности и качества моделей и алгоритмов идентификации и интерпретации результатов газогидродинамических исследований интеллектуальных скважин, позволяющих определять фильтрационные параметры и энергетическое состояние пластов, число режимов и время завершения исследований в процессе их проведения.

Приведенные модели и алгоритмы интерпретации комбинированных газодинамических исследований четырех газовых скважин, оснащенных стационарными информационно измерительными системами, показывают, что разработанные модели и алгоритмы адаптивной идентификации и интерпретации с учетом и корректировкой дополнительных априорных данных и экспертных оценок параметров пластов позволяют: 
1) обеспечить устойчивость и повысить точность оценок проницаемости, пъезопроводности пласта, пластового давления, скин-фактора скважины;

2) сократить число режимов исследований по индикаторной кривой до трех циклов и время проведения комбинированных газогидродинамических исследований;

\section{СПИСОК ЛИТЕРАТУРЫ}

1. Алиев 3.С., Зотова Г.А. Инструкция по комплексному исследованию газовых и газоконденсатных пластов и скважин. М.: Недра, 1980. - 301 c.

2. Давлетбаев А.Я., Исламов Р.Р., Иващенко Д.С. Особенности построения индикаторных диаграмм при газодинамических исследованиях скважин, проведенных ускоренными методами // Нефтяное хозяйство. - 2015. - № 11. - С. 36-40.

3. Rawlins E.L., Schellhardt M.A. Back pressure Data on Natural Gas Wells and their Application to Production Practices. Monograph Series, vol. 7. USBM, 1936.

4. Cullender M.H. The isochronal performance method of determining the flow characteristics of gas well // Trans. AIME. 1955. - V. 204. - P. 137-142.

5. Katz D.L. et al. Handbook of Natural gas Engineering. - New York City: McGraw Hill Book Co., Inc., 1959. - 705 p.

6. Horne R.N. Modern well test analysis. A computer-aided approach. - Palo Alto, California: Petroway, inc., 2000 - 257 p.

7. A new method of deliverability prediction by steady point in gas wells / A. Mu, Q. Liu, H. Tao, Zh. Du, K. Li, K. Xiao // Petroleum. - 2016. - V. 2. - P. 183-188.

8. Mahnaz H., Shahab G. A new fast approach for well production prediction in gas-condensate reservoirs // Journal of Petroleum Science and Engineering. - 2018. - V. 160. - P. 47-59.

9. Integrated study of gas condensate reservoir characterization through pressure transient analysis / J. Li, G. Zhao, X. Jia, W. Yuan // Journal of Natural Gas Science and Engineering. 2017. - V. 46. - P. 160-171

10. Нгуен Т.Х.Ф., Сергеев В.Л. Метод идентификации индикаторной кривой при интерпретации результатов газодинамических исследований скважин // Известия Томского политехнического университета. Инжиниринг георесурсов. - 2015. - Т. 326. № $12 .-$ C. $54-59$.

11. Nguyen T.H.P., Sergeev V.L., Strelnikova A.B. Adaptive interpretation of gas well deliverability tests // IOP Conference Series: Earth and Environmental Science. - 2016. - V. 43. - № 1.
3) обрабатывать короткие недовосстановленные кривые восстановления давления.

Показана целесообразность использования рекуррентных алгоритмов адаптивной идентификации с учетом априорной информации, что позволяет обрабатывать большие объемы данных забойного давления, сократить вычислительные затраты.

URL: http://iopscience.iop.org/1755-1315/43/1/012015 (дата обращения 11.10.2016).

12. Сергеев В.Л., Ву К.Д. К оптимизации адаптивных алгоритмов идентификации и интерпретации гидродинамических исследований с учетом влияния ствола скважины // Доклады Томского государственного университета систем управления и радиоэлектроники. - 2016. - Т. 19. - № 3. - С. 98-102.

13. Sergeev V.L., Kalayda V.T., Polishchuk V.I. Models and Algorithms of Non-Stationary Signal Identification in Conditions of Uncertainty // International Siberian Conference on Control and Communications, SIBCON Proceedings, 2016. URL: https://ieeexplore.ieee.org/document/7491708/ (дата обращения 16.06.2016).

14. Houpert A. On the Flow of Gases in Porous Media // Revue de L'Institut Francais du Petrole. - 1959. - V. 11. - № 14. P. $1468-1684$.

15. Al-Hussainy R., Ramey Jr H.J., Crawford P.B. The Flow of Real Gases through Porous Media // J Pet Technol. - 1966. - V. 5. № $18 .-$ P. 624-636

16. Пантелеев А.В., Летова Т.А. Методы оптимизации в примерах и задачах. - М.: Высшая школа, 2002. - 544 с.

17. Koshel R.J. Enhancement of the downhill simplex method of optimization // International Optical Design Conference. - 2002. V. 4832. - P. 270-282.

18. Vau B., Bourles H. Generalized convergence conditions of the parameter adaptation algorithm in discrete-time recursive identification and adaptive control // Automatica. - 2018. - V. 92. P. 109-114.

19. Li J., Zheng Y., Lin Zh. Recursive identification of time-varying systems: Self-tuning and matrix RLS algorithms // Systems \& Control Letters. - 2014. - V. 66. - P. 104-110.

20. Tikhonov A., Arsenin V. Solutions of Ill-Posed Problems. - New York: Wiley, 1977. $-258 \mathrm{p}$.

Поступила 01.03.2018 г.

\section{Информация об авторах}

Сергеев В.Л., доктор технических наук, профессор отделения нефтегазового дела Инженерной школы природных ресурсов Национального исследовательского Томского политехнического университета.

Нгуен T.X.Ф., аспирант отделения нефтегазового дела Инженерной школы природных ресурсов Национального исследовательского Томского политехнического университета. 


\title{
MODELS AND ALGORITHMS FOR ADAPTIVE INTERPRETATION OF COMBINED WELL TEST DATA OF INTELLIGENT WELLS
}

\author{
Viktor L. Sergeev', \\ SergeevVL@ignd.tpu.ru \\ Phuong T.H. Nguyen', \\ nguyenphuongtpu1512@gmail.com \\ 1 National Research Tomsk Polytechnic University, \\ 30, Lenin Avenue, Tomsk, 634050, Russia.
}

The urgency of the discussed issue is caused by the need to develop models and adaptive algorithms to analyze combined well test data of intelligent wells equipped with permanent downhole gauges which allow determining the parameters of reservoirs and wells when testing in real time.

The main aim of the study is to increase the efficiency and quality of integrated systems of models (rates, bottomhole pressures, additional a priori data and expert estimates of gas reservoir parameters) with variable time-dependent parameters and algorithms for adaptive identification and interpretation of combined well test data at steady and unsteady state of well test by indicator diagram and pressure building up curve.

The methods used in the study are the theoretical and practical developments in well testing, system analysis, system identification, optimization of functions and linear algebra. For analyzing the accuracy and stability of models and algorithms of identification and interpretation the authors used the field data of well test by indicator diagram and pressure building up curve, expert estimates of reservoir pressure and filtration parameters.

Results. The analysis of the efficiency and quality of models and algorithms of identification and interpretation on the examples of processing oil and gas well test data by indicator diagram and pressure building up curve has shown that integrated systems of models and algorithms of adaptive identification and interpretation allow: determining filtration parameters and the energy state of reservoirs and wells, the number of test stages and the completion time of the test within data input process in real time; providing stability and improving accuracy of estimates of permeability, reservoir conductivity, reservoir pressure, well skin factor due to the use and correction of additional a priori data and expert reservoir pressure and reservoir filtration parameters; analyzing short poorly reconstructed bottomhole pressure building up curves after well shut in.

Key words:

System analysis, identification, interpretation, well test, indicator diagram, pressure building up curve, integrated systems of model, a priori information, gas condensate fields.

\section{REFERENCES}

1. Aliev Z.S., Zotov G.A. Instruktsia po kompleksnomu issledovaniyu gazovykh i gazokondensatnykh plastov $i$ skvazhin [Instruction of complex well test of gas and gas-condensate fields]. Moscow, Nedra publ., 1980. $301 \mathrm{p}$.

2. Davletbaev A.Ya., Islamov R.R., Ivashchenko D.C. Features of IPR curves in case of modified gas well test. Neftyanoe khozyaistvo, 2015, no. 11, pp. 36-40. In Rus.

3. Rawlins E.L., Schellhardt M.A. Back pressure Data on Natural Gas Wells and Their Application to Production Practices. Monograph Series, vol. 7. USBM, 1936

4. Cullender M.H. The isochronal performance method of determining the flow characteristics of gas well. Trans. AIME, 1955, vol. 204, pp. 137-142.

5. Katz D.L. Handbook of Natural gas Engineering. New York City, McGraw Hill Book Co., Inc., 1959. 705 p.

6. Horne R.N. Modern well test analysis. A computer-aided approach. Palo Alto, California, Petroway, inc., 2000. 257 p.

7. Mu A., Liu Q., Tao H., Du Zh., Li K., Xiao K. A new method of deliverability prediction by steady point in gas wells. Petroleum, 2016, vol. 2, pp. 183-188.

8. Mahnaz H., Shahab G. A new fast approach for well production prediction in gas-condensate reservoirs. Journal of Petroleum Science and Engineering, 2018, vol. 160, pp. 47-59.

9. Li J., Zhao G., Jia X., Yuan W. Integrated study of gas condensate reservoir characterization through pressure transient analysis. Journal of Natural Gas Science and Engineering, 2017, vol. 46, pp. 160-171.
10. Nguyen T.H.P., Sergeev V.L. Identification method of indicator diagram by interpreting the measured results of gas-dynamic well testing. Bulletin of the Tomsk Polytechnic University. Geo Assets Engineering, 2015, vol. 326, no. 12, pp. 54-59. In Rus.

11. Nguyen T.H.P., Sergeev V.L., Strelnikova A.B. Adaptive interpretation of gas well deliverability tests. IOP Conference Series: Earth and Environmental Science, 2016, vol. 43, no. 1. Available at: http://iopscience.iop.org/1755-1315/43/1/012015 (accessed 11 October 2016).

12. Sergeev V.L., Vu Q.D. Optimization of adaptive algorithms for identification and interpretation of hydrodynamics research considering wellbore storage. Doklady Tomskovo gosudarsvennovo universiteta system upravleniya i radioelektroniki, 2016, vol. 19, no. 3, pp. 98-102. In Rus.

13. Sergeev V.L., Kalayda V.T., Polishchuk V.I. Models and Algorithms of Non-Stationary Signal Identification in Conditions of Uncertainty. International Siberian Conference on Control and Communications, SIBCON Proceedings, 2016. Available at: https://ieeexplore.ieee.org/document/7491708/ (accessed 16 June 2016).

14. Houpert A. On the Flow of Gases in Porous Media. Revue de L'Institut Francais du Petrole, 1959, vol. 11, no. 14, pp. 1468-1684.

15. Al-Hussainy R., Ramey Jr H.J., Crawford P.B. The Flow of Real Gases through Porous Media. J Pet Technol, 1966, vol. 5, no. 18, pp. 624-636.

16. Panteleev A.V., Letova T.A. Metody optimizatsii v primerakh $i$ zadachakh [Optimization methods in examples and problems]. Moscow, Vyshaya shkola Publ., 2002. 544 p. 
17. Koshel R.J. Enhancement of the downhill simplex method of optimization. International Optical Design Conference, 2002, vol. 4832, pp. 270-282.

18. Vau B., Bourles H. Generalized convergence conditions of the parameter adaptation algorithm in discrete-time recursive identification and adaptive control. Automatica, 2018, vol. 92, pp. 109-114.
19. Li J., Zheng Y., Lin Zh. Recursive identification of time-varying systems: Self-tuning and matrix RLS algorithms. Systems \& Control Letters, 2014, vol. 66, pp. 104-110.

20. Tikhonov A., Arsenin V. Solutions of Ill-Posed Problems. Wiley, New York, 1977. 258 p.

\section{Information about the authors}

Viktor L. Sergeev, Dr. Sc., professor, National Research Tomsk Polytechnic University.

Phuong T.H. Nguyen, postgraduate, National Research Tomsk Polytechnic University. 